\title{
Electromagnetic Shock Waves from Transmission Lines
}

\author{
D. Grischkowsky, I. N. Duling, III, J. C. Chen, and C.-C. Chi \\ IBM Watson Research Center, Yorktown Heights, New York 10598 \\ (Received 15 June 1987)
}

\begin{abstract}
We have observed subpicosecond electrical pulses to propagate on 5- $\mu \mathrm{m}$ coplanar transmission lines at velocities faster than the phase velocity in the underlying dielectric. This situation produces an electromagnetic shock wave in a manner similar to Cherenkov radiation and electro-optic Cherenkov radiation. Using time-domain spectroscopy, we have measured the strong frequency-dependent loss of energy in the propagating electrical pulse due to this radiation.
\end{abstract}

PACS numbers: 41.10.-j, 03.50.-z, 84.40.-x

Electromagnetic radiation is emitted when electric charges are accelerated, while the uniform motion of electric charges does not cause the emission of radiation. However, Cherenkov has shown that, when charges move faster than the phase velocity for electromagnetic radiation in a material, radiation is emitted as an electromagnetic shock wave. ${ }^{1}$ The initial analysis of this effect was for electric monopoles, but the physical picture holds true for higher-order moments as well.

Auston has recently demonstrated that it is possible to produce an electric dipole moving faster than the appropriate phase velocity in a dielectric, and thereby to produce an electromagnetic shock wave. ${ }^{2,3}$ This dipole, which is caused by an ultrashort laser pulse driving the optical rectification effect in a nonlinear dielectric material, has the same spatial distribution as the laser pulse. When the group velocity of light (and, consequently, the speed of the dipole) is faster than the phase velocity for terahertz radiation, an electromagnetic shock wave is produced. This situation has been verified in detail in Auston's experiments and theoretical analyses.

In this paper we describe another very general physical situation which can also yield emission of electromagnetic shock-wave radiation. This case involves the propagation of ultrashort electrical pulses on a metallic transmission line on the surface of a semi-infinite dielectric substrate. Associated with these propagating electrical pulses are electric multipoles determined by the number of metal lines making up the transmission line and the characteristics of the propagating mode. For example, with two metal lines the TEM mode has an electric dipole moment and a consequent dipolar field distribution, while for three lines a quadrupole moment is possible. When the dielectric constant is greater than unity, the group velocity for electric pulses propagating on these lines (and consequently the associated multipoles) will be faster than the phase velocity in the dielectric. Thus in the dielectric we have the situation shown in Fig. 1(a), which includes radiation of an electromagnetic shock wave in the form of a Cherenkov-type cone.

In this Letter we present time-domain measurements of the attenuation versus frequency for the spectral com- ponents of the propagating electrical pulse, due to the radiation of the electromagnetic shock wave. This loss is so severe that after propagating only $1 \mathrm{~mm}$, power at 0.8 $\mathrm{THz}$ is reduced to $1 / e$ of its original value. Previous frequency-domain studies have calculated the radiative loss for single-frequency transmission on coplanar metal lines on dielectrics. ${ }^{4}$ These results have been confirmed by experimental measurements up to $1 \mathrm{GHz},{ }^{4}$ where the radiation loss is only $10^{-9}$ of that reported here. Our observed frequency-dependent absorption remains in
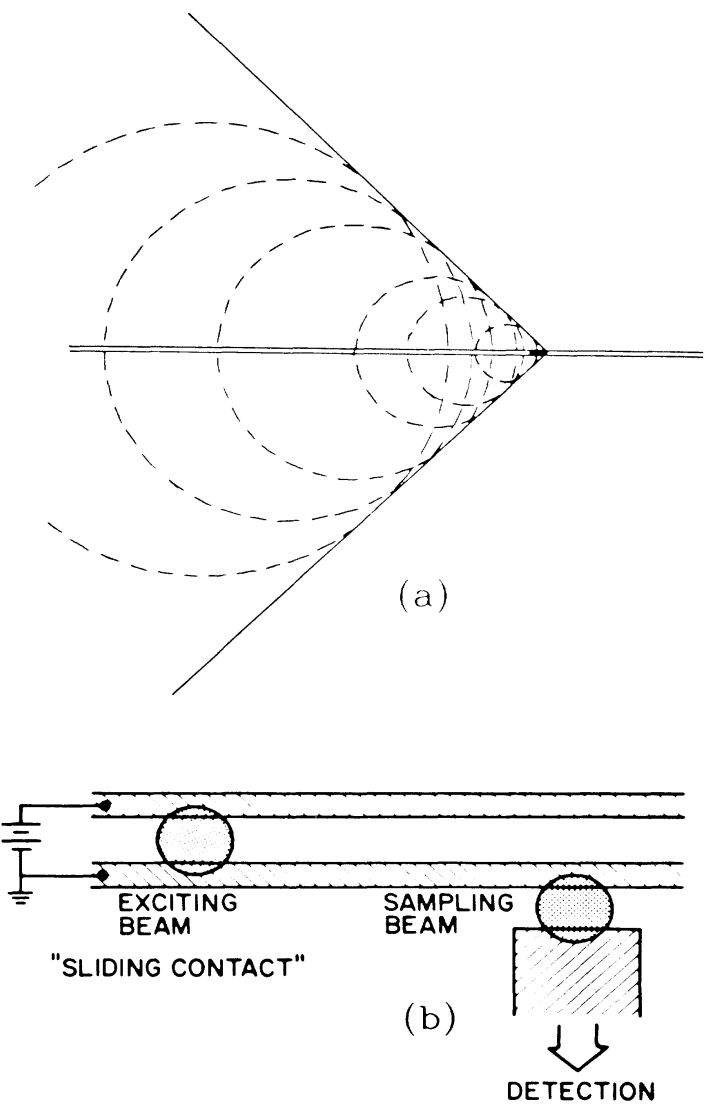

FIG. 1. (a) Cherenkov cone in the dielectric half space; (b) experimental geometry. 
good agreement with this calculation, even though our spectrum extends to $1 \mathrm{THz}$. At these high frequencies, the time-domain Cherenkov picture provides a simple intuitive description of the generation of the electromagnetic shock wave and of the radiation process associated with the propagating ultrashort electrical pulse.

The experimental situation shown in Fig. 1(b) is similar to that used previously. ${ }^{5}$ Here the subpicosecond electrical pulses are obtained by photoconductive shorting of the charged coplanar transmission line with 70fsec pulses from a colliding-pulse mode-locked dye laser. This method of pulse generation ensures a pure dipolar pulse. The resulting electrical pulses were measured by a fast photoconductive switch, driven by a time-delayed beam of the same $70-f$ sec laser pulses, which connected the transmission line to an electrical probe. In order to measure propagation effects, the excitation point (sliding contact) was moved variable distances away from the sampling photoconductive switch.

The 20-mm-long transmission line had a design impedance of $150 \Omega$ and consisted of two parallel $5-\mu \mathrm{m}$ wide, $0.5-\mu \mathrm{m}$-thick aluminum lines separated from each other by $15 \mu \mathrm{m}$. The measured dc resistance of a single $5-\mu \mathrm{m}$ line was $200 \Omega$. The transmission line was fabricated on an undoped silicon-on-sapphire wafer, which was then heavily implanted with $\mathrm{O}^{+}$ions to ensure the required short carrier lifetime. ${ }^{6}$ The measurements were made with the standard excite-and-probe arrangement for the beams of optical pulses.

In Fig. 2(a), we show the measured subpicosecond electrical pulse. For this result the spatial separation between the exciting and sampling beams was approximately $50 \mu \mathrm{m}$, while the laser spot diameters were 15 $\mu \mathrm{m}$. When the sliding contact was moved $6 \mathrm{~mm}$ from the optical sampling gap, we observed the pulse propagation effects shown in Fig. 2(b). The Fourier analyses of these pulses are shown in Fig. 2(c), where it is seen that the amplitude spectrum of the initial pulse extends to beyond $1 \mathrm{THz}$ and that the transmitted pulse has suffered a significant frequency-dependent loss. From these spectra it is straightforward to obtain the absorption coefficient as a function of frequency, as shown in Fig. 2(d). For some time, previous to this paper, we had considered this absorption to be due to the aluminum lines.

In order to test this assumption, we reduced the resistivity of the Al lines by approximately 12 times by cooling the transmission line to liquid-helium temperatures. Instead of the expected reduction in the absorption coefficient by at least $\sqrt{12}$ due to the consequent reduction in the surface resistance controlled by the frequency-dependent skin effect, we obtained the results shown in Fig. 3. The initial pulse looks almost identical to the room-temperature result. The unexpected feature was that the propagated pulse, illustrated in Fig. 3(b), broadened considerably and developed a slight ringing. In addition, as shown in Fig. 3(c), the Fourier spectra of the input and propagated pulses at $2.5 \mathrm{~K}$ look very similar to the roomtemperature results. This comparison is made more precise when the absorption coefficient is plotted [Fig. 3(d)].
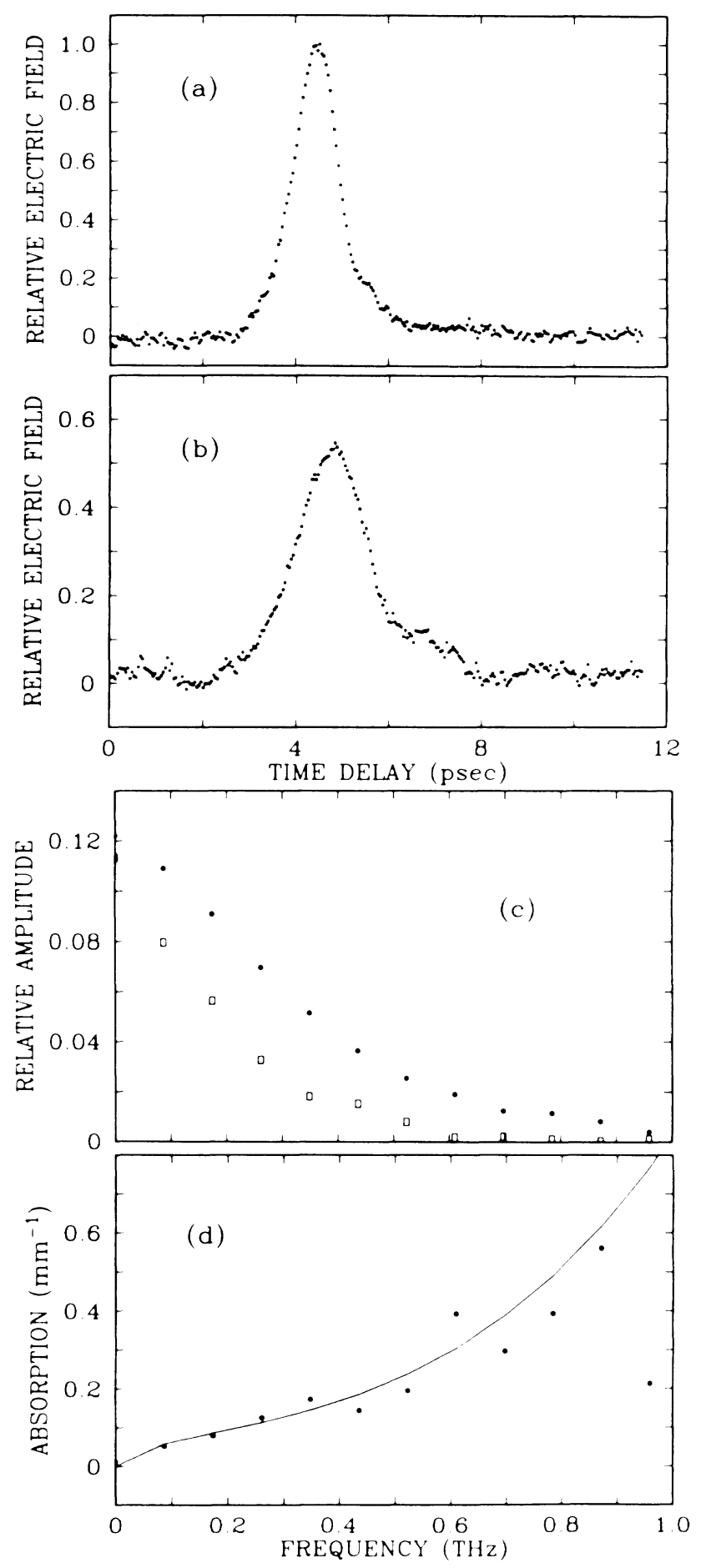

FIG. 2. Room temperature: (a) measured input pulse; (b) measured pulse after propagating $6 \mathrm{~mm}$ on the transmission line; (c) amplitude spectrum of input pulse (dots) compared with propagated pulse (squares); (d) amplitude absorption coefficient $\alpha$ from (c) (dots) compared with theory (solid line). 


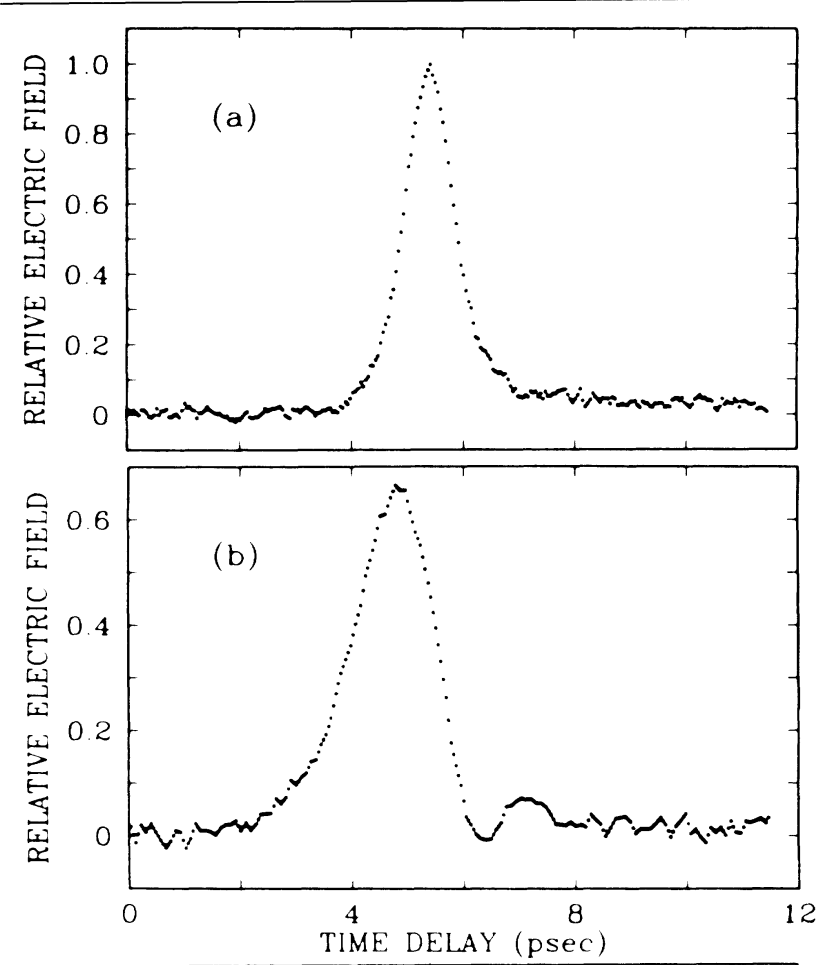

insignificant ${ }^{7}$ and cannot explain the large absorption observed. The same is true for the sapphire substrate whose absorption coefficient in this frequency range is also insignificant. ${ }^{8}$ Therefore, the apparent absorption must be due to radiation by the moving electric dipole associated with the electrical pulse. This pulse is produced by the transfer of approximately 2000 electrons between the two lines at the generation site. Consequently, two dipoles with charges of $1000 e$ and separations of $15 \mu \mathrm{m}$ move down opposite directions of the line at the group velocity. By measuring the total optical delay required for the sampling pulses, we determined that the electrical pulses (dipole moments) propagate on the line with the group velocity of $v_{g}=c / 2.45$. This velocity is significantly faster than the phase velocity of approximately $c / 3.3$ for terahertz radiation in sapphire.

The above measured value for $v_{g}$ is to be compared with that obtained from the relationship $v_{g}=c /\left[\frac{1}{2}(1+\right.$ $\epsilon)]^{1 / 2}$, which applies for our dielectric half-space geometry in the quasistatic approximation. ${ }^{9}$ The lowfrequency dielectric constants $\epsilon$ of sapphire are 9.4 for the ordinary ray and 11.6 for the extraordinary ray. ${ }^{8}$ These values give the corresponding group velocities of $c / 2.28$ and $c / 2.51$, which bracket our measured value of $c / 2.45$ obtained on sapphire of unknown orientation. This good agreement confirms the first-order validity of the quasistatic approximation and allows the calculation of the deviations from the TEM mode responsible for the radiative loss.

The solution for the radiation loss at frequency $\omega$ from a coplanar line on a dielectric is given by ${ }^{4}$

$$
\alpha=\left(\frac{\pi}{2}\right)^{5} \frac{\sqrt{2}(3-\sqrt{8})(1-1 / \epsilon)^{2} W^{2}}{\lambda_{d}^{3} K(k) K^{\prime}(k)}(1+1 / \epsilon)^{1 / 2} .
$$

For Eq. (1), $\lambda_{d}$ is the wavelength in the dielectric; $W=25 \mu \mathrm{m}$ is the width of the transmission line; $k=0.6$ is the ratio of the separation of the two lines to the total width; $K(k)$ and $K^{\prime}(k)$ are standard tabulated elliptic integrals. ${ }^{10}$

Evaluating Eq. (1) for our conditions, we obtain the amplitude absorption coefficient to be $\alpha=\left(0.4 \mathrm{~mm}^{-1}\right)$ $\times f^{3}$, where $f$ is the frequency in terahertz. This result is to be compared with the measurements in Figs. 2(d) and 3(d). The solid line fitted to the data in Fig. 2(d) is given by the relationship $\alpha=\left(0.2 \mathrm{~mm}^{-1}\right) f^{1 / 2}+(0.65$ $\left.\mathrm{mm}^{-1}\right) f^{3}$. The first term gives the frequency-dependent loss due to the resistive skin effect for the $\mathrm{Al}$ lines, while the second term with the $f^{3}$ dependence describes the loss due to the radiation. In Fig. 3(d), the solid line fitted to the measured absorption is again the sum of two terms, $\alpha=\left(0.06 \mathrm{~mm}^{-1}\right) f^{1 / 2}+\left(0.65 \mathrm{~mm}^{-1}\right) f^{3}$. It is to be noticed that the radiative term, as expected, remained the same, while the resistive-loss term decreased by approximately $1 / \sqrt{12}$, corresponding to the reduction of 12 
in the resistance of the lines. The measured loss due to the radiation is approximately 1.5 times the calculated value; we consider this good agreement and especially the cubic frequency dependence to confirm definitely the nature of the effect.

As an additional test of our conclusions, we performed the same measurements on a transmission line composed of $2.5-\mu \mathrm{m}$-wide lines separated by $5 \mu \mathrm{m}$. Here, because $W$ was reduced by 2.5 times compared with the measurements of Figs. 2 and 3, and in agreement with the prediction of Eq. (1), the radiative loss was negligible. The measured absorption was well described by the singleterm expression $\alpha=\left(0.4 \mathrm{~mm}^{-1}\right) f^{1 / 2}$ at room temperature. When the sample was cooled to liquid-helium temperatures, the resistivity of the lines dropped by 4 times and the absorption was fitted by the function $\alpha=(0.2$ $\left.\mathrm{mm}^{-1}\right) f^{1 / 2}$, which agrees with the expected reduction of $\frac{1}{2}$ due to the factor-of -4 decrease in resistivity.

This research was partially supported by the U.S. Office of Naval Research.
${ }^{1}$ J. V. Jelley, Cherenkov Radiation and Its Applications (Pergamon, New York, 1958).

${ }^{2}$ D. H. Auston, Appl. Phys. Lett. 43, 713 (1983).

${ }^{3}$ D. A. Kleinman and D. H. Auston, IEEE J. Quantum Electron. 20, 964 (1984).

${ }^{4}$ D. B. Rutledge, D. P. Neikirk, and D. P. Kasilingham, in Infrared and Millimeter Waves, edited by $\mathrm{K}$. J. Button (Academic, New York, 1983), Vol. 10, Pt. II.

${ }^{5}$ M. B. Ketchen, D. Grischkowsky, T. C. Chen, C.-C. Chi, I. N. Duling, III, N. J. Halas, J-M. Halbout, J. A. Kash, and G. P. Li, Appl. Phys. Lett. 48, 751 (1986).

${ }^{6}$ F. E. Doany, D. Grischkowsky, and C.-C. Chi, Appl. Phys. Lett. 50, 460 (1987).

${ }^{7}$ C. M. Randall and R. D. Rawcliffe, Appl. Opt. 6, 1889 (1967).

${ }^{8}$ E. E. Russell and E. E. Bell, J. Opt. Soc. Am. 57, 543 (1967).

${ }^{9}$ R. E. Collin, Field Theory of Guided Waves (McGrawHill, New York, 1960).

${ }^{10}$ Handbook of Mathematical Functions, edited by M. Abramowitz and I. A. Stegun, National Bureau of Standards Applied Mathematics Series No. 55 (U.S. GPO, Washington, D.C., 1971); see p. 608. 